\title{
Gestión de costos de las actividades en el sector metalmecánico de la región zuliana*
}

\author{
Rodríguez Medina, Guillermo** \\ Rodríguez Castro, Belkis*** \\ Chirinos González, Alira ${ }^{* * * *}$ \\ Meleán Romero, Rosana ${ }^{\star * * *}$
}

\section{Resumen}

El presente artículo tiene como objetivo analizar la gestión de costos de las actividades del sector metalmecánico de la región zuliana, debido a la importancia capital que tiene en el desarrollo de la principal industria Venezolana, como es petróleos de Venezuela, S.A. (PDVSA), con la finalidad de potenciar el éxito en la toma de decisiones gerenciales, y se sustentó en autores como: Kaplan y Cooper (1999), Brimson (1997), Lorino (1993), Horngren et al. (2007), Hansen y Mowen (2007), entre otros. El tipo de investigación fue descriptiva, de diseño no experimental, transeccional y de campo, mediante un cuestionario dirigido a los analistas de costos, de las empresas metalmecánicas fabricantes de bienes de capital de los municipios Maracaibo y San Francisco del Estado Zulia, registradas en el Directorio de la Cámara Petrolera Venezolana. Los resultados indican que muy pocas empresas realizan un análisis de las actividades, pero no se profundizan en sus propiedades, por lo que se concluye que los costos indirectos de fabricación o carga fabril no se gestionan de acuerdo al contexto contemporáneo.

Palabras clave: Gestión de costos, actividades, carga fabril, sector metalmecánico.

\section{Recibido: 21-07-08 Aceptado: 09-03-09}

* Este artículo es resultado del proyecto "Gestión de costos organizacionales en el sector meltalmecánico de la Región Zuliana", financiado por el Consejo de Desarrollo Científico y Humanístico (CONDES), de la Universidad del Zulia (L.U.Z).

** Doctor en Ciencias Mención Gerencia, Profesor Titular L.U.Z. Investigador del Centro de Estudios de la Empresa (CEE). Acreditado en el Programa de Promoción al Investigador (PPI), Nivel II, E-mail: rodrigueguillermo@gmail.com; grodriguezm@cantv.net.

*** Mg. en Gerencia de Empresas, Mención: Gerencia Financiera. Profesora de la URBE. Coinvestigadora Auxiliar del CEE de L.U.Z. PPI Nivel Candidato. E-mail: belkisr@msn.com

**** MSc. en Gerencia de Empresas, Mención: Gerencia de Operaciones. Profesoras Agregadas de L.U.Z. Investigadoras del CEE. PPI Nivel I. E-mails: alirachirinos@gmail.com; rosaname- 


\section{Cost Management for Activities in the Metallurgical Sector of the Zulia Region}

\section{Resumen}

The purpose of this article is to analyze cost management for activities in the metallurgical sector in the Zulia region, in order to strengthen success in managerial decision making, due to the capital importance this sector has in developing the principal Venezuelan industry, the Venezuelan Petroleum Company (Petróleos de Venezuela, S. A.) (PDVSA). The study is supported by the works of authors such as: Kaplan and Cooper (1999), Brimson (1997), Lorino (1993), Horngren et al (2007), Hansen and Mowen (2007), among others. The research was of a descriptive type with a nonexperimental, trans-sectional, field design, using a questionnaire directed to cost analysts at the metallurgical companies that manufacture capital goods in the municipalities of Maracaibo and San Francisco in the State of Zulia, registered in the Directory of the Venezuelan Petroleum Chamber. Results indicate that very few companies perform activity analyses, and do not go deeply into their properties. Conclusions are that the indirect manufacturing costs or manufacturing load is not managed according to the contemporary context.

Key words: Cost management, activities, manufacturing load, metallurgical sector.

\section{Introducción}

El sector metalmecánico ocupa un prestigioso lugar dentro de la economía nacional, particularmente en la región $\mathrm{Zu}$ liana, debido a que forma parte de la cadena de suministros de Petróleos de Venezuela, S.A. (PDVSA), industria pilar de la economía venezolana, por lo que el presente artículo pretende analizar la gestión de costos de las actividades en las empresas metalmecánicas fabricantes de bienes de capital, registradas en el directorio de la Cámara Petrolera de Venezuela (CPV) capítulo Zulia, en los Municipios Maracaibo y San Francisco.

De acuerdo con Gibas (2005), presidente de Asociación de la Industria Metalmecánica y Metalúrgica Venezolana, el sector creció entre el $10 \%$ y el $12 \%$ el primer semestre del año 2005 en relación con el mismo lapso de 2004, lo cual le permitió generar 20 mil empleos entre di- rectos e indirectos. Por otro lado, Touma (2006), director de los empresarios en Corpozulia, manifiesta que la capacidad de producción de las empresas manufactureras de la región zuliana está entre un $70 \%$ y $80 \%$ de su capacidad instalada, y fueron asignados los primeros cincuenta millones de dólares, con un interés aproximado del $8 \%$, para la recuperación de las empresas zulianas, principalmente para los sectores plásticos, camaroneros, industriales y metalmecánicos.

Esta situación plantea la gran importancia del sector metalmecánico en la región zuliana y la recuperación del mismo, después de los sucesos acaecidos a finales del año 2002 y principios del 2003 , donde la producción petrolera bajó casi a cero, de allí lo fundamental de este trabajo de investigación, para analizar la gestión de costos de las actividades.

Dado que existe en el sector meltalmecánico gran competencia, tanto a nivel 
nacional como internacional, en este mundo globalizado, es imprescindible que las empresas realicen una planificación y un control de los costos de manufactura, a través de las actividades para crecer tanto en el sector como en el mercado, tomando en cuenta el desarrollo de la región donde se encuentren inmersas.

En el contexto actual, debido a la internacionalización de la economía, las empresas metalmecánicas se han visto en la necesidad de ejercer una mejor gestión de sus costos, particularmente tomando en cuenta el análisis de las actividades, para el mejor desarrollo del proceso de producción, que permita una mejor planificación, ejecución y control de los costos.

El presente trabajo, fue un estudio de investigación descriptiva, de acuerdo a Hernández et al. (2006), quienes afirman que estos "miden, evalúan o recolectan datos sobre diversos conceptos (variables), aspectos, dimensiones o componentes del fenómeno a investigar". El diseño de la investigación fué no experimental, dado que no se manipularon intencionalmente las variables, pues, se observaron los fenómenos tal como se dan en la realidad para su análisis, además, es del tipo transeccional, dado que los datos fueron recolectados en un momento dado y no a través de diferentes periodos o puntos en las dimensiones del tiempo, y de campo por cuanto la fuente primaria se obtuvo directamente de las empresas objeto de estudio.

La población de acuerdo a Parra (2006), estuvo integrada por las mediciones $u$ observaciones de interés en la investigación, a las catorce (14) empresas metalmecánicas fabricantes de bienes de capital, según el directorio de la Cámara Petrolera Venezolana, del año 2005, en Ios municipios Maracaibo y San Francisco de la región zuliana; trabajándose con censo y no con muestra.

La recopilación de los datos se realizó mediante la aplicación de un cuestionario a los analistas de costos de producción. La data recabada se organizó y se proceso a través del paquete estadístico SPSS versión 10.0 , para sus análisis y discusión de los resultados.

\section{Procesos: algunas definiciones}

Antes de abordar las actividades, como tal, es conveniente en primera instancia tratar lo referente a los procesos, para lo cual se contrastan diferentes posiciones; así se tiene que Lorino (1993:36), establece que, "se llamarán procesos a los conjuntos de actividades destinadas a la consecución de un objetivo global, a una salida global, tanto material como inmaterial"; para Álvarez et al. (1996:307), definen el proceso, “... como un conjunto coordinado de tareas que desembocan en la obtención de un producto o de un servicio específico".

Por otro lado Fernández et al. (2006:9) manifiestan que, "el proceso de producción es un conjunto de actividades mediante las cuales uno o varios factores productivos se transforman en productos. La transformación crea riqueza, es decir, añade valor a los componentes o input adquiridos por la empresa", mientras que Rodríguez et al. (2007), expresan que los procesos, "están compuestos por un conjunto de actividades relacionadas entre sí, generalmente en forma transversal, 
transcendiendo diferentes funciones para obtener una finalidad común a la organización". Además, hay que hacer un diagrama de flujo de los procesos, que según García (1995:156), "es una representación gráfica de la secuencia de actividades o pasos que ocurren en un proceso $o$ en un procedimiento, identificándolos mediante símbolos de acuerdo con su naturaleza; incluye, además, toda la información que se considera necesaria para el análisis...".

Con las apreciaciones anteriores se tiene que los procesos en las organizaciones están integrados por una serie de actividades que operan bajo unos procedimientos coordinados en forma sistemática, que trascienden el objetivo funcional con el norte de alcanzar el global de la organización, es decir, el proceso agrupa a las actividades en forma tal, que permite la obtención de una finalidad común. De tal manera que los procesos abarcan una serie de actividades transfuncionales, que buscan permanentemente la mejora continua, que es el corazón de la gestión moderna de costos de toda organización, particularmente las manufactureras, para obtener una salida global, ya sea para un cliente interno o externo.

\section{Gestión de costos de las actividades}

Es preciso en primera instancia indicar que Pacheco et al. (2002:8) señalan que la "gestión debe incluir un conjunto de dimensiones, que involucran una estrategia y unos medios para alcanzar los objetivos de la empresa". Por otro lado, Taiichi Ohno, citado por los mismos autores, define a la gestión integral como "el sistema que desarrolla la habilidad humana hasta la plena capacidad a fin de mejorar la creatividad y la prosperidad, utilizando el utillaje y las maquinas correctamente y eliminando cualquier costo improductivo".

El concepto de costo, de acuerdo a Hansen y Mowen (2007:35), "es el efectivo o un valor equivalente de efectivo sacrificado por productos y servicios que se espera que aporten un beneficio presente o futuro a una organización", mientras que para Mallo et al. (2000:33), el concepto de costo tiene varias connotaciones de acuerdo al interés de cada disciplina, así por ejemplo, "el concepto económico de costo se ha utilizado en dos versiones generales: la primera en sentido de consumo o sacrificio de recursos de factores productivos, y la segunda en el sentido de costo alternativo o de oportunidad".

De tal manera que el costo desde el punto de vista contable es el sacrificio necesario de recursos financieros o no, para producir $o$ adquirir bienes tangibles $o$ intangibles que generen beneficios presentes o futuros, y cuando esto ocurre el costo expira y se convierte en gasto, y desde el punto de vista económico el costo también puede ser de oportunidad, el cual mide el beneficio perdido por no haber sido empleado en la mejor alternativa.

Por otro lado, la actividad es la parte medular para la gestión de costos y en términos globales de toda la organización, con énfasis particular en esta investigación en el proceso productivo de las empresas metalmecánicas productoras de bienes de capital.

De acuerdo a Sáez et al. (1993:187), "una actividad es un conjunto 
Gestión de costos de las actividades en el sector metalmecánico de la región zuliana Rodríguez, Guillermo; Rodríguez, Belkis; Chirinos, Alira y Meleán, Rosana

de tareas o actos imputables a un grupo de personas o a una persona, a un grupo de máquinas o a una máquina, y relacionadas con un ámbito preciso de la empresa". Para Castelló y Lizcano (1994), citado por Álvarez et al. (1996:308) "es un conjunto de actuaciones o de tareas que tienen como objetivo la atribución, al menos a corto plazo, de un valor añadido a un objeto (producto o proceso), o al menos permitir añadir este valor, bajo la perspectiva del cliente o usuario del mismo"; mientras Brimson (1997:26) manifiesta que, "una actividad describe lo que una empresa hace, la forma en que el tiempo se consume y las salidas (outputs) de los procesos", y Lorino (1993:36) indica que, "las actividades es todo lo que las personas de la empresa realizan hora tras hora y día tras día".

Como se aprecia en las definiciones anteriores, las actividades están referidas al conjunto de tareas realizadas por una o varias personas, que partiendo del requerimiento y el consumo de una serie de recursos se obtiene un resultado determinado; en el sector metalmecánico objeto de estudio, ellas son las que influyen y participan directamente, junto con la mano de obra directa, en la transformación de la materia prima, teniendo relaciones de interdependencia, que al estar encadenadas en forma ordenada y articulada conforman el o los procesos.

La contabilidad de costos es parte fundamental de la de gestión, ya que ésta viene dada por todo un sistema que involucra a las diferentes áreas funcionales de la organización. En tal sentido, la gestión de costos de las actividades se refiere al establecimiento de las estrategias, que se llevan a cabo a través de las accio- nes que permitan escudriñar la compleja interacción de ellas que se dan en el proceso productivo del sector metalmecáni$\mathrm{co}$; en aras de generar las exigencias informativas que el entorno, tanto interno como externo, requiere para la mejor toma de decisiones gerenciales.

Asimismo, para determinar el costo de las actividades con mayor exactitud, indudablemente hay que conocer y comprender la intríngulis que encierra dicho término, para lo cual es necesario desagregarlo, en aspectos como la identificación de ellas, el diccionario, el alcance, la unidad de análisis, la clasificación, los atributos, la capacidad, la jerarquía, los inductores y el sistema de información.

La identificación de las actividades, consiste en su determinación a través de verbos, como por ejemplo fresar, tornear, entre otros, los cuales pueden realizarse utilizando a los trabajadores de primera línea del proceso productivo, a la vez que permite establecer la relación entre funciones, procesos y de ellas mismas.

En lo referente al diccionario de las actividades, no es más que después de identificarlas se hace un listado de las mismas, donde se indique la o las tareas que la integran y su conceptualización; al respecto Kaplan y Cooper (1999:94) manifiestan que, "la identificación de las actividades culmina con la construcción de un diccionario de actividades que nombra y define cada actividad realizada en las instalaciones de producción".

En cuanto al alcance, este está dado por la delimitación de las mismas en cuanto a donde comienza y donde termina, para su definición y análisis, sobre el particular Brimson (1997:110) manifiesta que este alcance, "limita el abanico de ac- 
tividades especificadas en el análisis de actividades de manera que la información pueda ser recogida eficientemente"; es decir, establece hasta donde se desea llegar y permite identificar las tareas innecesarias dentro de ella.

La unidad de análisis de las actividades, permite realizar el diagnóstico y la mejora potencial de la misma, a través de un proyecto creativo, en el que estén involucrados todos los actores participantes para garantizar el éxito, y será validado por los propios interesados, para poder monitorear el comportamiento de los costos y el rendimiento de los recursos que son los inputs, para lo cual es necesario cuantificar la ejecución a través, de una unidad que permita medir el nivel de ella y determinar el resultado logrado por dicha actividad, es decir, el output, pero primeramente se debe determinar la unidad que se va a analizar, por ejemplo un centro de costos, y luego las actividades que hay dentro de ella.

De acuerdo a Brimson (1997:124), en cuando a la clasificación, manifiesta que, "cada actividad debe ser clasificada como primaria o secundaria", entendiendo por primaria aquella cuyo resultado $u$ output se utiliza fuera de la unidad de análisis y la secundaria son aquellas que se dan dentro de la misma unidad para apoyar a las primarias. Según, Lorino (1993) indica que pueden clasificarse por su naturaleza, su activación o su destino, donde las de según su naturaleza las subclasifica en de diseño, de realización, de mantenimiento y discrecionales, las de activación en autónomas y ordenadas, y las de destino en primarias y secundarias.
Para Álvarez et al. (1996) las clasifica por funciones, por su naturaleza, por su relación con el objetivo de costo y por su aportación a la generación de valor. Donde las funcionales se refieren a aquellas que están relacionadas al área de la organización, por su naturaleza las subclasifica en fundamentales y discrecionales, por su relación con el objetivo de costo en las relacionadas con el volumen de producción, con los procesos productivos, con el sostenimiento y con infraestructura, en cuanto a la generación de valor se tienen las que añaden y las que no agregan valor.

Rodríguez et al. (2002:143), afirman que las actividades generadoras de valor o no, se originan de la concepción de cadena de valor de Porter (1980), y manifiestan que:

...lo que se busca en desagregar todas las actividades tanto internas como externas a la organización, ya que cada una de ellas es un eslabón de dicha cadena, para hacer énfasis en las relevantes que agregan valor al producto, retomándolas, reforzándolas y mejorándolas, debido a que serán percibidas por los consumidores con un mejoramiento continuo de la calidad, y eliminar así aquellas actividades superfluas que no añaden ningún valor al bien, y lo único que hacen es agregar costo al mismo.

De las clasificaciones anteriores, si bien es cierto que Álvarez et al. (1996) se apoyan en Lorino (1993), las profundiza y toma en cuenta otros aspectos relacionados con las funciones y su aportación en la generación de valor, sin embargo, deja de lado las de destino que son de mucha 
importancia para la toma de decisiones gerenciales e incluso hay autores que solo toman en cuenta éstas, por considerarlas más relevantes.

Por último, los atributos de las actividades están referidos a las características que ella encierra, donde se especifiquen los recursos que utiliza (inputs), el control que de ellas se ejerce, el objetivo y las restricciones, la eficiencia con que se manejan los insumos cuando se ejecuta dicha actividad, además de los recursos físicos, el costo de los mismos, la calidad, el plazo en que se realiza y la entrega del producto (output) a la siguiente etapa del proceso, tal como se aprecia en el Diagrama 1.

La especificación de los atributos facilita la recopilación y el seguimiento del comportamiento de los costos; Kaplan y Cooper (1999:101) manifiestan que "Ios atributos son esquemas de codificación asociados con cada actividad que facilitan que se informe de los costes de la misma", mientras que Álvarez et al. (1996:308) señalan que, para identificar las actividades será importante tener en cuenta los atributos que suelen caracterizarla, y de entre los que podemos citar los siguientes:

a) La actividad es repetitiva, en cuanto al proceso que desarrolla.

b) Consume recursos de coste y tiempo.

c) Tiende a obtener un output (productos/servicios).

d) Está condicionada por variables tales como: tiempos limitados, cumplimientos de calidad, frecuencia, etc.

e) Una actividad en un determinado contexto puede constituir una simple tarea....

Para Holst y Savage (2002:13), citado en Player y Lacerda, indican que, "los atributos cuantifican aspectos diferentes de procesos de negocios, proporcionando puntos de vista múltiples con los cuales enfocar, priorizar, analizar y medir esfuerzos y resultados organizacionales". Los atributos, pues, permiten informar si las actividades agregan valor,

\section{Diagrama 1 \\ Descripción de la Actividad}

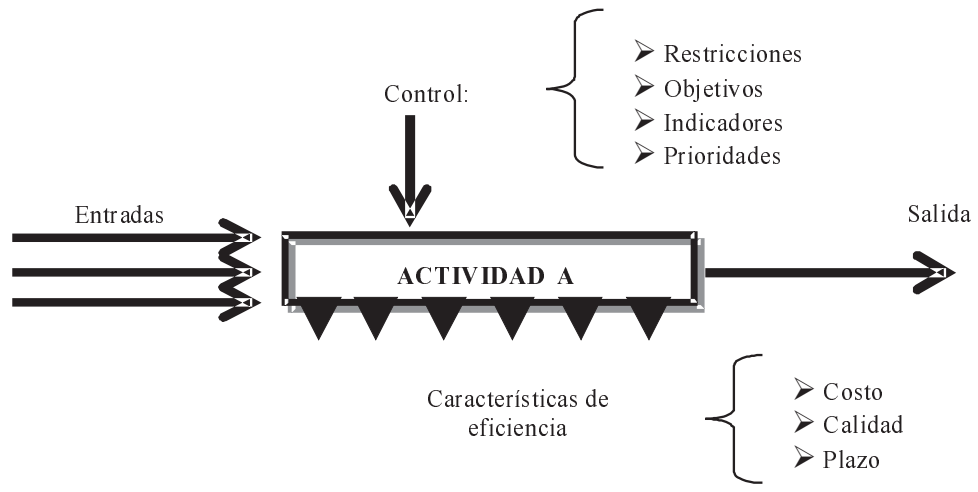

Fuente: Lorino (1993:60). 
y sí se realizan con eficiencia para apoyar a los factores clave de éxito.

Los atributos son determinados de acuerdo a la complejidad de la organización, pero además de medir la eficiencia del uso de los recursos, es conveniente saber la variabilidad de los costos para tener una visión de los que son fijos y variables en el corto plazo en sus operaciones.

La capacidad permite la asignación de los costos indirectos de fabricación fijos (CIFF), a través del inductor de costo, para determinar la tasa de aplicación, y ésta se determina dividiendo el presupuesto de CIFF entre la capacidad representada por el nivel del denominador, por lo que la determinación de la capacidad es clave para el cálculo de costos y la toma de decisiones gerenciales, particularmente para los costos basados en actividades (C.B.A.).

De acuerdo a Horngren et al. (2007:309-10) clasifica a la capacidad en: teórica, práctica, normal y del presupuesto maestro.

La capacidad teórica se refiere al nivel del denominador para producir con plena eficiencia todo el tiempo, lo cual es utópica ya que no considera ningún tipo de mantenimiento, ni de interrupciones; la práctica es la capacidad que toma en cuenta las interrupciones operacionales inevitables, por lo tanto el nivel del denominador es menor que el de la teórica; la capacidad normal además de las interrupciones normales, toma en cuenta la demanda promedio de los clientes en periodo de dos a tres años y el nivel del denominador puede ser menor al de la práctica; por último se tiene la capacidad presupuestada que se basa en aquella que se va a utilizar el próximo año, de tal ma- nera que el nivel del denominador puede diferir al de la normal. Al respecto Kaplan y Cooper (1999:119) manifiestan que, "medir, crear y gestionar la capacidad no utilizada es la clave del calculo de costos ABC".

Para una mejor toma de decisiones gerenciales es conveniente que el factor de costo de los CIFF, se determine basado en la capacidad práctica como nivel del denominador, debido a que evitará establecer nuevos costos unitarios cuando cambie la demanda esperada, y se enfrenta con mayor eficacia a la competencia, porque permite a la gerencia determinar la capacidad de producción no utilizada, y el costo de esta no forma parte del costo del producto.

Las cualidades que enmarcan a las actividades, son consideraciones importantes para determinar con mayor exactitud los costos que se incurren en ellas, y que son las que constituyen la parte medular del costeo basado en actividades, para lo cual también es necesario profundizar el tema sobre la jerarquización de las mismas, con la finalidad de crear conjuntos de costos y de esta manera facilitar la determinación tanto del costo total como del unitario de los productos, particularmente a lo referente a los costos indirectos de fabricación, dentro de su estructura.

En relación a la jerarquización de actividades, para una mejor gestión, se establecen de acuerdo a lo contemplado en Kaplan y Cooper (1999), Horngren et al. (2007), Gayle (1999), Mallo et al. (2000), Sáez et al. (1993), Hansen y Mowen (2007) y Barfield et al. (2005), donde se jerarquizan, de la siguiente manera: Las de nivel unitario, se dan cada 
Gestión de costos de las actividades en el sector metalmecánico de la región zuliana Rodríguez, Guillermo; Rodríguez, Belkis; Chirinos, Alira y Meleán, Rosana

vez que produce una unidad, por tanto se agrupan en los elementos del costo, y particularmente los costos de materiales y mano de obra directa, las de nivel de lote, cada vez que se fabrica uno de ellos, las de nivel de línea de productos, son las que se ejecutan en cada una de las líneas de los procesos productivos y se incrementan en la medida que aumente la diversidad de productos, por tanto los costos se agrupan de esta manera, y por último las de nivel de instalación, que son las que apoyan los procesos de manufactura en general de una fábrica.

Además, de la jerarquización es necesario el establecimiento de los inductores de costos en cada uno de los niveles tanto de entrada como de salida a la actividad.

Los costos se ocasionan porque siempre hay una factor que impulsa a que este se origine y es precisamente esto lo que se denomina inductor de costo, que no es más que una variable que permite la vinculación causal del consumo de los recursos por las actividades y las de ésta con los objetos de costos.

Sobre este particular Brimson (1997:149) manifiesta que, "una relación causal existe cuando se puede demostrar que un factor de producción es consumido directamente por una actividad"; Lorino (1993:83) señala que "la identificación de los inductores es un procedimiento clásico de análisis causa/efectos", mientras que Sank y Govindarajan (1995:199) indican que, "... el costo es causado o manejado, por muchos factores que están interrelacionados en formas complejas".

Las diferentes definiciones mencionadas anteriormente vienen a confir- mar que el inductor de costo, es el factor que hace que el costo se incurra, que bien puede ser llamado conductor, generador o causal de costo.

Los inductores de costos se clasifican de acuerdo a Riley (1987), citado por Shank y Govindarajan (1995:26-27), en estructurales y de ejecución, mientras que Kaplan y Cooper (1999: 104-105) lo establecen en inductores de transacción, de duración y de intensidad.

Dentro de este contexto los inductores clasificados por Kaplan y Cooper (1999), están enmarcados dentro de los de ejecución establecidos por Riley (1987) debido a que ellos reflejan la habilidad de la empresa y permiten realizar las mejoras continuas dentro de los procesos, particularmente los productivos, para llevar con éxito la determinación del costo de las actividades, donde el de transacción está referido a la frecuencia con que se realiza una actividad, el de duración representa la cantidad de tiempo necesario para desarrollarla y el de intensidad establece una imputación directa de los recursos utilizados en cada actividad; de los tres el más exacto es este último y el menos es el de transacción.

En cuanto a los inductores estructurales, estos no indican una medida de rendimiento de los procesos sino que están referidos a la estructura económica de la organización, como por ejemplo la economía de escala y la complejidad, entre otras.

El sistema de información integrado juega un papel fundamental en el éxito de la gestión de costos en las actividades, tal como lo afirman Rodríguez et al. (2005:107), cuando señalan que es "la conjunción de elementos como el recurso 
humano, el hardware, el software que trabajan en forma armónica, organizada, sincronizada e integrada para procesar datos, ordenándolos, transformándolos y analizándolos, a través de los diferentes subsistemas organizacionales".

Actualmente existe en el mercado una gran variedad de software que permiten capturar la información requerida para la determinación de los costos de las actividades, tal como lo afirman Player y Marx (2002: 34), quienes manifiestan que, "ahora viene la nueva ola en el desarrollo de ERP (Enterprise resourse planning) a medida, que las compañías construyan sistemas de análisis, pronósticos y otros sistemas complementarios para reducir costos y mejorar el servicio al cliente".

Las consideraciones anteriores permiten afirmar que la gestión de costo de las actividades busca la sinergia de los objetivos de la organización y el mejor desarrollo eficiente y eficaz de los recursos que son consumidos por todas y cada una de las actividades, que conforman los procesos de la empresa.

\section{Análisis y discusión de los resultados}

En este punto se analizan e interpretan los resultados obtenidos en las catorce (14) empresas, a quienes se les suministró un instrumento para medir las variables objeto de estudio. En primer lugar, el $78,6 \%$ de ellas tienen identificadas bien sus actividades, sin embargo, solamente el $35,7 \%$ elabora el diccionario de las mismas, lo que indica que no se aprovecha la fortaleza de haberlas identificado, tal como lo establece Kaplan y
Cooper (1999), cuando afirman que la identificación de las actividades culmina con la construcción de un diccionario que nombra y define todas las que se realizan en las instalaciones de producción.

En cuanto al alcance, y a la unidad de análisis se observó que el $84,6 \%$ determina el alcance, sin embargo de ese total el $57,1 \%$ determina la unidad de análisis, lo que indica que no se utilizan bien estas herramientas gerenciales para garantizar el éxito de la gestión, ya que no se profundiza en las mismas; además, a pesar de que existe un alto porcentaje $(84,6 \%)$ que establece el alcance, no determinan las tareas innecesarias dentro de ellas, tal como lo indica Brimson (1997), en la descripción de las mismas e incluso se demuestra poca conceptualización sobre este particular.

En relación a la pregunta sobre la clasificación de las actividades, donde se suministró varias alternativas se evidenció que el $57,1 \%$ diferencia las primarias de las secundarias, el $50 \%$ también las clasifica por funciones, y el 14,2\% también las hace por la generación de valor, mientras que el $42,8 \%$ no realiza ningún tipo de clasificación. Se observa que no se profundiza en la clasificación, pues, las más usuales son las referentes a primarias y secundarias, le sigue la clasificación por funciones, mientras que solo dos empresas las hace por las que agregan y no añaden valor, y seis no hacen ninguna clasificación. Esta situación está en contraposición con lo establecido por Lorino (1993) y Álvarez et al. (1996), sobre la tipología, para una mejor comprensión y manejo de la gestión de costo de las actividades. 
Gestión de costos de las actividades en el sector metalmecánico de la región zuliana Rodríguez, Guillermo; Rodríguez, Belkis; Chirinos, Alira y Meleán, Rosana

Con respecto a los atributos, el $42,9 \%$ de las 14 empresas toman en cuenta la eficiencia, el 35,7\% de la población seleccionó el consumo de recursos y el $28,6 \%$ del total señaló la capacidad, sin embargo, cinco (5) de las catorce (14) empresas estudiadas, o sea, el $35,7 \%$ no toman en cuenta ningún tipo de atributo, lo que está en contraposición con lo señalado por Lorino (1993), Kaplan y Cooper (1999), Holst y Savage (2002), entre otros, quienes indican que las especificaciones de los atributos facilitan la cuantificación de los resultados de las actividades (output). Así mismo, las empresas que respondieron la capacidad como atributo, no especificaron si se referían a la teórica, práctica, normal o presupuestada, lo que significa que no se está claro sobre este asunto.

Sobre la jerarquización, se evidenció que solamente el $21,4 \%$ considera el nivel unitario, el $7,1 \%$ lo hace a nivel de línea de producto, mientras que el $71,5 \%$ respondió desconocer esta jerarquización, sin embargo, todas controlan lo referente a la materia prima y a la mano de obra directa, desconociendo que estos elementos forman parte del nivel unitario, tal como lo indican Horngren et al. (2007), Hansen y Mowen (2007) y Barfield et al. (2005).

Referente a la clasificación de los inductores de costo se encontró que el $21,4 \%$ utiliza el de transacción, el $7 \%$ el de intensidad, los cuales según Kaplan y Cooper (1999), el primero está referido a la frecuencia con que se realizan las actividades y el segundo establece la imputación directa de los recursos utilizados en cada una de ellas, mientras que el $71,4 \%$ respondió no conocer estos tipos de inductores, de igual manera la clasificación que hace Shank y Govindarajan (1995), en inductores estructurales y de ejecución.

Por otro lado, de las catorce (14) empresas estudiadas, el $21,4 \%$ utiliza el costeo real, el $14,2 \%$ el costeo normal y $64,3 \%$ el costeo estándar, utilizando pocos inductores de costos y los determinan en función del concepto tradicional, es decir, basado en el costo de la mano de obra directa y en horas hombres.

Esta situación hallada indica que las empresas examinadas no realizan un exhaustivo estudio de los inductores de costos, para determinar el verdadero factor que impulsa el costo, y en general del análisis de las actividades, a pesar que el $28,5 \%$ de ellas los costos indirectos de fabricación representan en promedio el $30 \%$ dentro de la estructura de costos, lo que significa la importancia de estos en el nuevo contexto dentro de la administración de costos, de acuerdo a lo indicado por Kaplan y Cooper (1999), Horngren et al. (2007), Sáez et al. (1993), entre otros.

Por otra parte, de las catorce (14) empresas objeto de estudio el $64,3 \%$ dispone de software para el manejo de los costos, sin embargo, no se aprovecha esta herramienta para utilizarlo como un sistema de información integral, que permita capturar toda la data requerida y relacionarla con las demás áreas funcionales de la organización, para que en forma oportuna y pertinente se pueda obtener toda la información correspondiente para la aplicación de la gestión de costos por actividades, de acuerdo a lo señalado por Rodríguez et al. (2005). 


\section{Conclusiones}

Las empresas objeto de estudio tienen bien definido los elementos del costo de los productos que elaboran, particularmente en cuanto a la materia prima y a la mano de obra directa, sin embargo, no así con los costos indirectos de fabricación (C.I.F.), que a pesar de tener identificadas sus actividades, que es una fortaleza para la determinación de los recursos que ellas consumen, tanto, en su aspecto físico como en sus costos, no aprovechan esta situación, lo cual limita la composición de los elementos que integran los C.I.F., y la realización de un verdadero análisis de sus actividades.

En cuanto al diccionario, no es llevado a cabo por las empresas objeto de estudio, a pesar de que las tienen identificadas, lo que impide conocer con exactitud la o las diferentes tareas que la componen, así como la interrelación entre funciones, procesos y las de ellas mismas entre sí.

El alcance, y la unidad de análisis, son determinados de manera superficial, lo que implica una limitación en el uso de éstas herramientas para la toma de decisiones en la gestión.

En cuanto a la clasificación y a los atributos, tampoco son utilizados de manera eficaz, solo aquellas que logran determinarlas lo hacen de manera parcial y ni siquiera tienen claro la capacidad utilizada en las operaciones, si está basada en la teórica, en la práctica, en la normal o la presupuestada, lo que está en contraposición con lo establecido en la fundamentación teórica, para el éxito de las empresas objeto de estudio, particular- mente para gestionar los costos indirectos de fabricación fijos.

Existe un desconocimiento sobre la jerarquización de los costos, sin embargo, acumulan estos solamente bajo el concepto tradicional, particularmente a lo referente a la materia prima y a la mano de obra directa, sin saber que ésta manera de agrupación es lo que se denomina nivel unitario. De igual forma sucede con los inductores de costos que no aplican esta categorización por su desconocimiento, a pesar de ser un factor importante para el establecimiento causal del consumo de los recursos.

De similar manera, un alto porcentaje de las empresas estudiadas tienen software para la captura de los datos y el manejo de los costos, pero no aprovechan esta situación para realizar un verdadero análisis de las actividades e integrar por medio del sistema de información todas las áreas funcionales de la organización, en aras de la obtención de mejores resultados; determinándose que las empresas objeto de estudio no gestionan los costos de las actividades de la mejor manera para garantizar el éxito en la toma de decisiones gerenciales.

\section{Referencias bibliográficas}

Álvarez, José.; Amat, Joan; Amat, Oriol; Balada, Tomás; Blanco, Felipe; Castelló, Emma; Lizcano, Jesús y Ripoll, Vicente (1996). Contabilidad de Gestión Avanzada. Planificación, Control y Experiencias Prácticas- Mc. GrawHill. España.

Barfield, Jesse; Raiborn, Cecily y Kinney, Michael. (2005). Contabilidad de Costos. Tradiciones e Innovacio- 
nes. Quinta Edición. Internacional Thomson Editores. México.

Brimson, James (1997). Contabilidad Por Actividades. Alfaomega grupo editor. México.

Fernández, Esteban; Avella, Lucia y Fernández, Marta (2006). Estrategia de Producción. Segunda edición. McGrawHill Interamericana. España.

García, Alfonso (1995). Productividad y Reducción de Costos. Para la pequeña y mediana industria. Editorial Trillas. México.

Gayle Rayburn (1999). Contabilidad y Administración De Costos. Sexta edición. Irwin Mc Graw Hill. México.

Gibas, A. (2005). Panorama, 2 de Septiembre de 2005. Sección Economía. Pg. 2-1. Maracaibo. Venezuela.

Hansen, Don y Mowen, Maryanne (2007). Administración de Costos. Contabilidad y Control. Internacional Thomson editores, S.A. de C.V. Quinta edición. México.

Hernández, Roberto; Fernández, Carlos y Baptista, Pilar (2006). Metodología de la Investigación. Cuarta edición. Mc Graw Hill. México.

Holst, Randolf y Savage, Robert (2002). Herramientas y Técnicas para Implementar la Administración Basada en Actividades. En Gerencia basada en actividades de Player, S. y Lacerda, R. Comp. Mc. Graw-Hill interamericana. Bogotá.

Horngren Charles, Foster, George. y Datar, Srikant (2007). Contabilidad de Costos: Un Enfoque Gerencial. Décima Edición. Prentice Hall. México.

Kaplan, Robert y Cooper, Robin (1999). Coste y Efecto. Cómo usar el ABC, el ABM y el ABB para mejorar la gestión, los procesos y la rentabilidad. Ediciones Gestión 2000. España.
Lorino, Philippe (1993). El Control de Gestión Estratégico. La Gestión por Actividades. Ediciones Alfaomega. México.

Mallo, Carlos; Kaplan, Robert, Meljem, Sylvia y Jiménez, Carlos (2000). Contabilidad de Costos y Estratégica de Gestión. Prentice Hall. España.

Pacheco, Juan; Castañeda, Widberto y Hernan, Carlos (2002). Indicadores Integrales de Gestión. Mc Graw Hill. Colombia

Parra, Javier (2006). Guía de Muestreo. Segunda edición. Colección FCES. Universidad del Zulia. Maracaibo. Venezuela.

Player, Steve y Marx, Charles (2002). Convergencia del Software Empresarial al Costeo basado en Actividades. En Gerencia basada en actividades de Player, S. y Lacerda, R. Comp. Mc Graw Hill. Bogotá.

Porter, Michael (1980). Estrategia Competitiva. Técnicas para el análisis de los sectores industriales y de la competencia. Editorial continental. México.

Rodríguez, Guillermo; Balestrini, Solange; Melean, Rosana y Rodríguez, Belkis (2002). Análisis Estratégico del Proceso Productivo en el Sector Industrial. Revista de Ciencias Sociales. Vol. VIII. No. 1. Universidad del Zulia. Maracaibo. Venezuela. Pg. 135-156.

Rodríguez, Guillermo; Chávez, Jorge; Rodríguez, Belkis y Chirinos, Alira (2007). Gestión de Costos de Producción en el Sector Metalmecánico de la Región Zuliana. Revista de Ciencias Sociales. Universidad delZulia. Maracaibo. Venezuela.

Rodríguez, Guillermo; Rodríguez, Belkis y Chirinos, Alira. (2005). Sistema de Información en la Gestión Presupuestaria Sector Pastas Alimenticias. Revista de Ciencias Sociales. Vol. XI, No. 1. Universidad del Zulia. Maracaibo. Venezuela. Pg. 105-117. 
Sáez, Ángel.; Fernández, Antonio y Gutiérrez, Gerardo (1993). Contabilidad de Costes y Contabilidad de Gestión. Volumen I. Mc Graw-Hill interamericana. España.

Shank, John y Govindarajan, Vijay (1995). Gerencia Estratégica de Costos. La
Nueva Herramienta para Desarrollar una Ventaja Competitiva. Editorial Norma. Colombia.

Touma, Bassan. (2006). Panorama, 11 de Septiembre de 2006. Sección Política, Pg. 2-1. Maracaibo. Venezuela. 